sex and/or receptive anal sex. These swabs are analysed using the Aptima Combo II platform, for Neisseria gonorrhoea (GC). Aim(s)/objectives With analysis costing $£ 6.20$ per swab we sought to explore cost effectiveness, review culture results and partner notification results.

Methods Inclusion criteria were heterosexual patients with exclusively extra-genital GC who did not present as a contact of GC. We performed a retrospective case note review of 54 sets of notes asserting symptom history, concurrent STI diagnosis, culture results and any positive contacts.

Results Over the year, a total of 13123 throat swabs were sent. There were 50 confirmed positive results giving swabs sent per positive result ratio of $262: 1$, or a cost of $£ 1624.40$ per positive result. For rectal swabs; a total of 1362 were sent. There were 4 positive results (all female) giving swabs sent per positive result ratio of $341: 1$, or a cost of $£ 2114.20$ per positive result. $2 \%$ of patients with a positive extra-genital swab result gave a history of throat or rectal symptoms. $18 \%$ had a concurrent STI diagnosis, $0 \%$ had a positive culture result from the same site. $6 \%$ had at least one subsequent positive contact, all of which were pharyngeal positive.

Discussion/conclusion Extra-genital testing has detected cases which would otherwise have been missed with purely genital screening. However numbers are too small to advocate a change in practice to routine extra-genital screening in all asymptomatic individuals.

\section{P29 AUDIT OF RE-TESTING AND REINFECTION IN LONDON MEN WHO HAVE SEX WITH MEN WITH ACUTE STIS IN A LARGE GUM OUTPATIENT CLINIC}

Laura Williamson*, Mauro Proserpio, Olamide Dosekun. Imperial College Healthcare NHS Trust, London, UK

\subsection{6/sextrans-2015-052126.73}

Background Men who have sex with men (MSM) in the UK are at relatively high risk of acquiring new STIs. The British Association of Sexual Health and HIV recommend active recall of MSM diagnosed with sexually transmitted infections (STIs) for retesting after 3 months.

Objectives An audit was undertaken to assess the incidence of bacterial STIs, and rates of re-screening and re-infection amongst MSM attending a large genitourinary (GU) outpatient clinic in London.

Methods A retrospective audit of GU coding data on MSM attendees aged $>18$ years between January and December 2014 was performed. Data was collected on patient demographics, STI tests performed and diagnoses.

Results 397 MSM were diagnosed with 826 new bacterial STIs during the audit period (762 STIs over 534 episodes occurred in the initial 9 month period). 145 (37\%) patients were HIV infected. In 98/534 (18\%) episodes, a repeat screen was performed within 3 months (excluding screening within the initial 6 weeks after an STI was diagnosed); in 21 (21\%) of these episodes, a further $1 \geq$ STI was diagnosed. Overall, the mean time to re-screening during the study period was 108 days (excluding initial 6 weeks; range 43-282). In 149/534 (28\%) of STI episodes, no repeat STI screen was performed within the period analysed.

Conclusion The incidence of STIs and re-infection in this high risk group is high, however prompt re-screening rates are low, highlighting the need for active recall. Routine 3 month text recall of MSM with an STI has since been implemented.

\section{P30 GONORRHOEA: A RISING TIDE}

Kanchana Seneviratne, Ruth Taylor, Sophia Farmilo, Shereen Munatsi, Ashini Fox*. Nottingham University Hospitals NHS Trust, Nottingham, UK

\subsection{6/sextrans-2015-052126.74}

Background The prevalence of gonorrhoea in England increased by $15 \%$ between 2012 and 2013. In contrast, there was a $62 \%$ rise in gonorrhoea in our local area in the same time period.

Aim To identify potential areas for management improvement that may help reduce infection rates.

Methods A retrospective case note review of positive patients between 1 st January and $30^{\text {th }}$ June 2013 was conducted. Positive agar-based gonococcal culture or BD ProbeTec ${ }^{\mathrm{TM}}$ GC $\mathrm{Q} x$ Amplified DNA Assay results were included.

Results The 201 individuals reviewed had a mean age of 24 (range 16-53). 53\% were male, 80\% Caucasian and 89\% heterosexual. There was no geographical postcode pattern seen. $100 \%$ resolution of infection at test of cure (TOC) was achieved in the $39 \%$ that attended. $10 \%$ TOC attendees became reinfected. 100\% received Partner Notification (PN), of whom $45 \%$ had contacts attending for treatment and 36\% declined to provide contact details.

Discussion Unlike the epidemic elsewhere in the UK, our outbreak is predominantly amongst male and female heterosexuals. As the majority were in the age range 16-25, targeted screening and health promotion could be delivered using the same resources as the National Chlamydia Screening Programme locally. TOC attendance was poor and the use of automatic text reminders and TOC postal kits maybe beneficial. The quality of information provided for PN can be improved with novel methods of non-standard PN. The high re-infection rate suggests a large reservoir of undiagnosed disease in our local population which needs addressing on a larger public health basis.

\section{P31 DIFFERENCES IN DISTRIBUTION OF PLANTAR SKIN RASH OF SECONDARY SYPHILIS AND KERATODERMA BLENORRHAGICA}

Johnny Boylan*, Peter Greenhouse. Bristol Sexual Health Centre, Bristol, UK

\subsection{6/sextrans-2015-052126.75}

Background/introduction Textbooks commonly assert that the most important cause of plantar skin rash is secondary syphilis $\left(2^{\circ} \mathrm{Syph}\right)$, but there are many other possible differentials, the principal alternative STI diagnosis being keratoderma blenorrhagica $(\mathrm{KB})$.

Aim(s)/objectives Observational study to quantify differences in distribution and character of plantar rash caused by $2^{\circ}$ Syph or KB.

Methods We sourced colour photographs of confirmed $2^{\circ} \mathrm{Syph}$ and $\mathrm{KB}$ from personal slide collections, illustrated textbooks and online academic websites, checked for evidence of correct diagnosis and showing at least $80 \%$ of the full plantar surface. Lesion distribution was categorised between either the weightbearing ball and heel or non-weightbearing arch of the foot with gradations shown in the Table 1.

Results We found 50 images of $2^{\circ}$ Syph and 25 of KB with reliably attributable clinical diagnoses. The overwhelming majority of $2^{\circ}$ Syph lesions were entirely or almost entirely (42/50) confined to the non-weightbearing arch of the foot: Conversely KS lesions were almost all (18/25) distributed over the thicker weightbearing areas. 